SHS Web of Conferences 6, 03015 (2014)

DOI: $10.1051 /$ shsconf / 20140603015

(C) Owned by the authors, published by EDP Sciences, 2014

\title{
Study on the Plastic Bottle Recycling Based on Evolution Tree for Technical System
}

\author{
Xiong Yuedong; Huang Huadong \\ Suzhou Institute of Industrial Technology, 215104 Suzhou Jiangsu, China
}

\begin{abstract}
Technical system theory of evolution tree was used in the study of the plastic bottle recycling, and established the evolutionary line of plastic bottle recycling on the basis of the analysis of plastic bottle recycling recovery evolution tree, and summed up a new smart plastic bottle recycling program. The new recovery recovers and smashes the plastic bottles through technical system, and communicates with users through automatically reward system and rewards the latter. The experimental prototype test results show: post treated fragments of plastic bottles are small, which are convenient to transport and take advantage of; the operation of recovery is easy, and the interface of man-machine interaction is friendly which is easy to expand functions.
\end{abstract}

Keywords. TRIZ; evolutionary tree; plastic bottle; recycling box; evolutionary line

\section{Introduction}

China has a huge population. Large amount of bottled water is consumed in the city every day. Randomly discarded plastic bottles can be seen everywhere, especially in impacted area and city's flourishing area. According to the data of China Packaging Federation, China annually produces 3 million tonnes plastic bottles, which will consume 18 million tonnes oil, which equivalent to yearly production of a medium-sized oil field. PET (polyethylene terephthalate) what plastic bottles used is also raw material of polyester staple fibre and filament. After simple treatment, recycled waste plastic bottles can be made into chemical fibre, and then made into clothing [1]. Moreover, the discarded plastic bottles can be processed into 'plastic rice' to reuse. Peoples have environmental conscious will throw waste plastic bottles into trash, otherwise throw away, thus caused environmental pollution. Therefore, in order to reduce plastic bottles environmental pollution, encourage peoples to make good use of renewable resource, and for the benefit of social, it is very necessary to research a kind of plastic bottle recycling unit which integrates recycling function, processing function and incentive function together. The paper studied plastic bottle recycling unit's evolution process based on TRIZ evolutionary tree theory, and designed a new generation plastic bottle recycling unit.

\section{Evolution tree}

TRIZ, meant inventive problem solving theory, is invention and innovation method developed on the basis of study a large amount of patented technologies, through decades of continuous effort by former soviet inventor Genrich·Altshuller and his invention team [2-4]. Evolutionary tree theory is created by Altshuller's disciples during the study of technical system evolution line. It is a new 
method for analysis and research of patent information. With evolution tree, new idea and technical solution can be gotten in product creative design. Evolutionary tree is built on the basis of technical system evolution line. It maps the information organizational structure of technical system development course, reflects technical system evolution essence and regularity. Technical system evolution tree can be constructed either manually or by computer. The two methods has no substantial difference, just the former method can give a panoramic view of the things studied, which is more convenient than the later. Evolution tree building approach is as follow:

- Determine main function of object studied. In order to build evolution tree of object which participant into accomplish one function, object function should be abstracted from its desired function when it is produced or be used, on account of that any function can be achieved by several different technical systems, and similarly any technical system can achieve several different functions.

- Gather information about similar objects. Simply describe every scheme of object analysed, especially the conversion essence of it, to find the initial solution of the object studied and the simplest plan in according to technical evolutionary sight.

- Choose main evolution line, that is, to construct the trunk of evolution tree. The main evolution line can be any evolution line of the evolutionary tree, but should be the most convenient to use and the most distinct of internal element transform, such as divide line of system and component, or 'single $\rightarrow$ double $\rightarrow$ multiple' system expansion line. Any of the evolution lines can turn to be evolution tree trunk. Branches are evolution lines derived from the trunk. Each point of the tree can construct multiple sequential evolution lines. Analyse each solutions on vertical trunk from the point of view whether there has resource to build its evolution line. For example, object that separated into two parts has two segment surfaces and an internal resource space. According to level demand of action to system, geometric evolution line, internal structure evolution line, and surface characteristic evolution line can be constructed once, then moveable connection is analysed and introduced in turn, and turn into more dynamic connection possibility.

- Build second level evolution line according to following rule: As much as possible to establish dynamic route of object conversion scheme. If no such possible, then firstly build the route which can apply dynamic resource, such as 'single $\rightarrow$ double $\rightarrow$ multiple' route, separating route and clipping route. To each step of evolutionary tree trunk, the possibility of using dynamic evolution route should be checked. To the initial solution of system, dynamic has to be achieved through change entire parameters of system. If dynamic is needed further enhancement, other resources are needed to be introduced.

- Examine possibility of construct second level evolution route which describes object shape transform and surface characteristic conversion and internal structure conversion. It includes geometric evolution, internal structure evolution, and surface characteristic evolution. In order to make the evolution tree structure best, add routes into the evolution tree only if those reflecting very important object conversion for latter analysis.

- Examine possibility of construct third level dynamic route after 'single $\rightarrow$ double $\rightarrow$ multiple' system evolution route, dividing route, and clipping route, then draw these routes on the corresponding feature point of the evolution tree.

- Construct improving controllable route after dynamic route.

- Complementary search for information, complement and confirm the structure of the evolution tree.

\section{Build evolution tree of environmental product}

According to the above steps, building evolution tree of plastic recycling unit, what should be pay attention is that the final state is not built at one blow. Using these methods, searching and handling information, the evolution tree will growth and evolution, just like a live tree, which change itself appearance, meanwhile bear fruits of new creative technology. Before collect information of recycling 
unit, main function of recycling unit and what role it played in the exercise of function should be make clear, The function what the recycling unit accomplishes can be described as plastic bottle recycling and treatment. When the function is finished, we can get plastic particles. Therefore, the initial definition of recycling unit is: recycling unit is used for recycling and dealing with plastic bottles.

To achieve any function, tool and action object is needed. While exercise recycling and handling plastic bottle function, what is tool and action object? Of course the plastic bottle being recycled is the action object, while device that processing plastic bottle is the tool.

When analyzing recycling unit, the action difference of its component while exercising function should be considered. Recycling unit is a complicate system, which composes of at least recycling device, processing device and auto controllable device. Furthermore, it also includes other elements, such as shell. Firstly, we should pay attention to the system component which acts as executive device, namely, handling plastic bottle device. After collected all kinds of recycling unit's information, we should determine the initial system solution, that is, from the technology evolution point of view the simplest solution. For recycling unit, we should make clear how to deal with plastic bottles in the easiest way.

After chosen the initial solution of recycling unit, we can enter the next phase of building evolution tree. What is important here is to determine which one will be the main route of evolution? Then take the route as the trunk.

Beforehand search can get multi-solution of plastic bottle recycling unit. Through analyse, we can choose following collector solution as key solutions:

- Basic container.

- Ordinary garbage box.

- Classification plastic bottle recycling unit.

- Multi-function plastic bottle recycling unit.

- Smart plastic bottle recycling unit..

Generally, any technical system during a long changing process can be described by 'system expansion-clipping (controllable)' evolution route. Therefore, this trend can be seen in recycling unit evolution tree, as shown in figure 1.

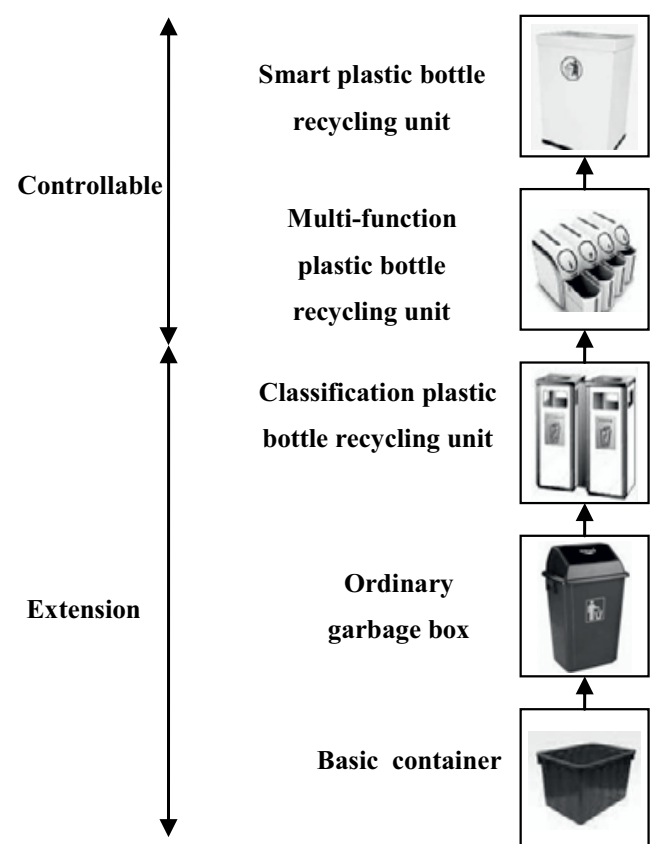

Figure 1. Collector extension - controlled evolution (evolution tree trunk). 
Expansion of recycling unit is by introducing new elements, such as applicability. Category recycling unit has achieved main useful function, yet the system needs further expansion. The next step of system expansion is achieved by the expansion of every part in system, that is, by construct controllable routes. Removing more shells from the system, remaining elements then grouped into one shell, thus produced multifunction plastic bottle recycling unit.

After evolution tree trunk is set up, and again information search is conducted, the result is a new, supplemental conversion solution. For example, in figure 1's smart plastic bottle recycling unit solution, according to object or material controllable route, new recycling unit solution is found and new tree branch content is added to the evolution tree, which is shown in figure 2.

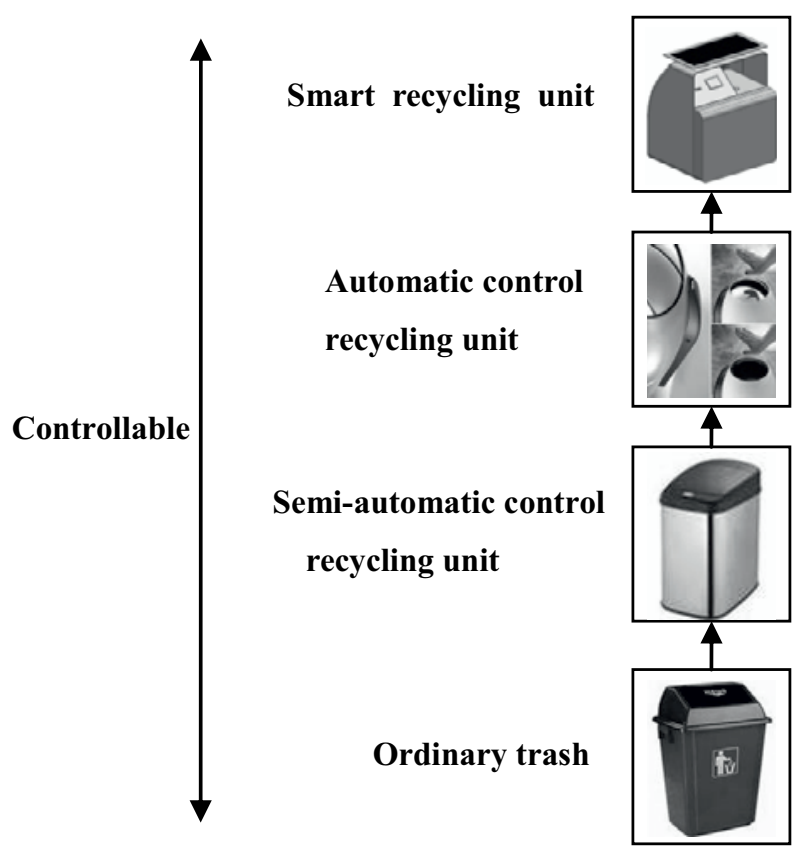

Figure 2. Collector controllable evolution (evolution tree branches).

\section{Innovative design of plastic bottle recycling unit}

If improve design product controllability according to the evolution route shown in Figure 2, then people will glad to throw used plastic bottles into the recycling unit, an then either solved recycling problem, or reach the aim of on-site treatment. For this purpose, on the basis of studied basic junk boxes, a new generation environmental plastic bottle recycling unit is put forward [5], which has reward mechanism, and integrated mechanical, electrical, embedded system, wireless network and computer server technology together. This kind of device is composed of three parts: mechanical crushing system, auto control system and wireless network controller system, its design scheme is shown in figure 3, and its work flow is shown in figure 4. After thrown a used plastic bottle into the slide way of recycling device, ARM controller system is stimulated by sensor, and then send signal to turn on the power of motor. The motor drive the mechanical system working, then knife tool subassembly grinds, washes, and dries the plastic bottle, reach the purpose of collection, storage, reusing. Meanwhile, after user inputted plastic bottle, they can obtain certain incentives by automatic reward system of recycling unit. When user insert special IC card into the recycling unit, its touch screen will show the amount of bottles inputed and the corresponding info of reward. What the user neeed to do is just follow the instruction and operation, meanwhile he can also choose to donate the prize gotten. All submitted data sended to remote server through wireless network for treatement. 


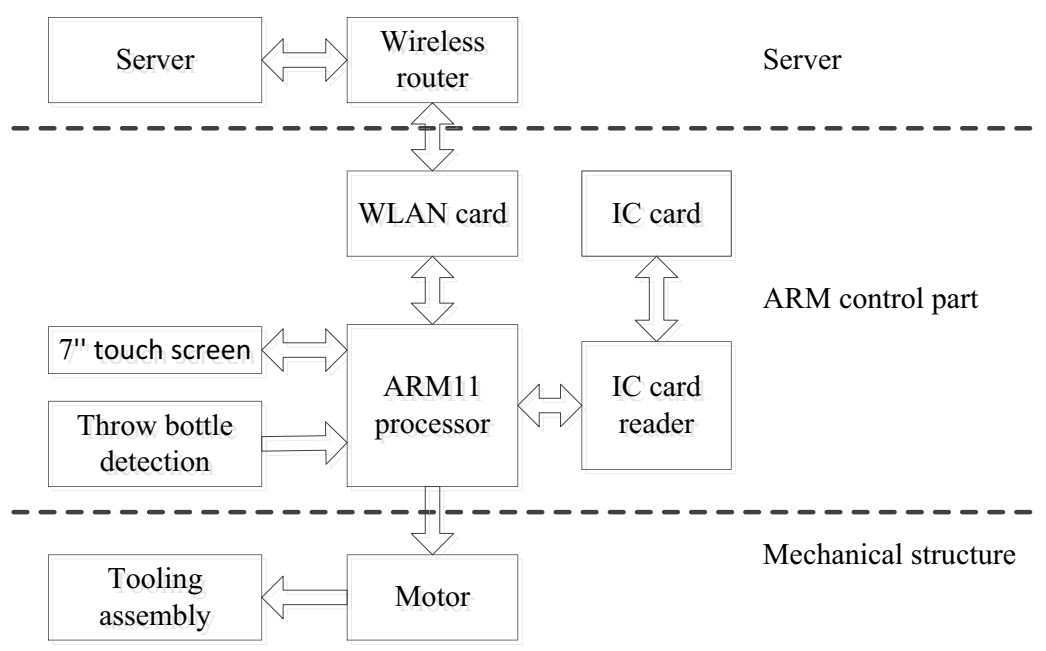

Figure 3. Design diagram of plastic bottles the smart collector system

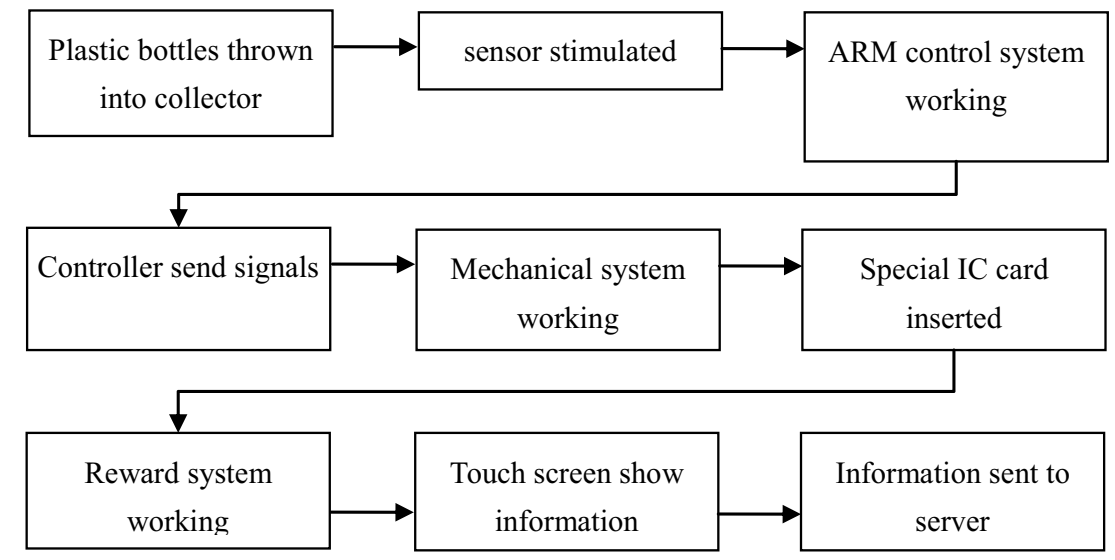

Figure 4. plastic bottles smart recovery workflow

\section{Overall structure of plastic bottle recycling unit}

The mechanical structure of the recycling unit is composed of motor, v-belt and cutting tool component, etc. [6]. After the motor switched on, it drives v-belt motion, and then drives belt pulley of cutting tool component motion. The belt pulley drives rotation of multiple high-speed steel knives set which is connected on the rotation axis of belt pulley. Through the shear force between motion knives and fixed knives, like scissors, the plastic bottle is crushed into tiny plastics, then is washed and dried. Figure 5 is the structure scheme of plastic bottle recycling unit. It expressed the appearance and internal structure of plastic smart recycling unit. The main parts of it is: top roof plate, touch screen, roof plate, input bottle door, v-belt, Crushing-washing-drying device, front door plate, junk box, solar energy plate, read card slot, ARM circuit board, plastic bottle pipeline, motor, etc. 


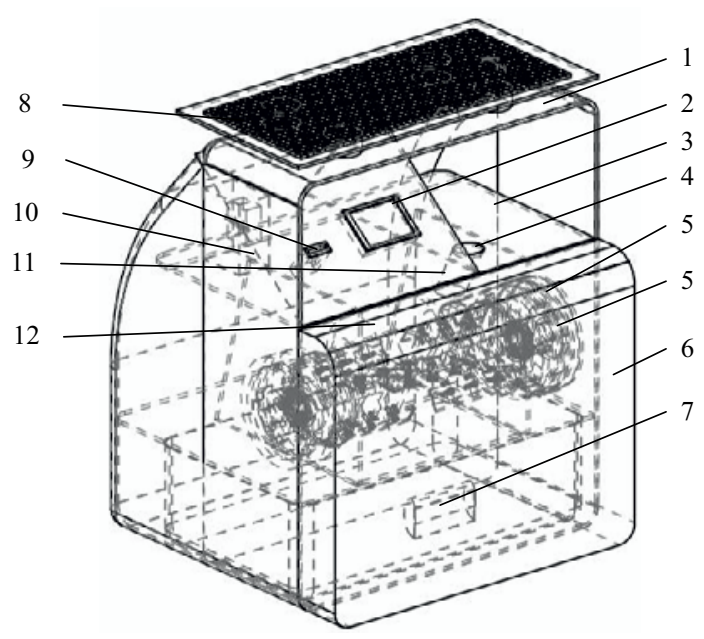

1. Top roof plate, 2. Touch screen, 3. Roof plate, 4. Input bottle door, 5. V-belt, 5. Crushing-washingdrying device, 6. Front door plate, 7. Junk box, 8. Solar energy plate, 9. Read card slot, 10.ARM circuit board, 11. Plastic pipeline, 12. Motor

Figure 5. New design of the plastic bottle recycling

\section{Conclusions}

The paper applied TRIZ theory's evolution tree analysis method, explored evolution route of plastic bottle recycling unit, putted forward recycle unit's creative design solution. Proto machine developed by this solution, has a good effect of on-site recycling and treatment of plastic bottle. The plastic particles are very small, either easy for transport, or good for following reuse. Moreover, the recycling unit reinforced the user's participant, autonomy, and interest. Using combined network information auto management, labor cost is significantly reduced. With reward mechanism, the recycling unit reinforced garbage classification thought, strengthened environmental protect awareness of people, brought forth new idea for advocate garbage recycling and for design corresponding creative products.

\section{References}

1. X. Du, Guangming Daily, 2011 (6)

2. H. Zhao, T. Jiang, Y. Li, M. Zhao, Chinese Journal of Engineering Design, 2012(19) : 75-80

3. F. Zhang, L. Zhang, P. Wang, Transactions of the Chinese Society for Agricultural Machinery, 2008(39): 116-119

4. P. Zhang, R. Tan, Transactions of the Chinese Society for Agricultural Machinery, 2010(41): $182-188$

5. Q. Zhang, L. Meng, Environmental Science \& Technology, 2010(33): 348-352

6. L. Chen, J. Yi, Industrial Safety and Environmental Protection, 2012(38): 12-13 